\title{
A new approach to assess function after sciatic nerve lesion in the mouse-Adaptation of the sciatic static index
}

\author{
Abrahão Fontes Baptista ${ }^{a, b, c}$, Joyce Rios de Souza Gomes ${ }^{\text {b }}$, Júlia Teixeria Oliveirab ${ }^{\text {, }}$ \\ Soraia Moreira Garzedim Santos ${ }^{\mathrm{b}}$, Marcos André Vannier-Santos ${ }^{\mathrm{c}}$, \\ Ana Maria Blanco Martinez ${ }^{\mathrm{a}, *}$ \\ ${ }^{a}$ Departamento de Histologia e Embriologia, Universidade Federal do Rio de Janeiro, Rio de Janeiro, Brazil \\ ${ }^{\mathrm{b}}$ Fundação Bahiana para Desenvolvimento das Ciências, Escola Bahiana de Medicina e Saúde Publica, Bahia, Brazil \\ ${ }^{\mathrm{c}}$ Centro de Pesquisas Gonçalo Moniz, Fundação Oswaldo Cruz, Bahia, Brazil \\ Received 24 March 2006; received in revised form 22 November 2006; accepted 27 November 2006
}

\begin{abstract}
Among the numerous ways of assessing regeneration after peripheral nerve lesions, the analysis of gait is one of the most important, because it shows the recovery of function, which is the ultimate goal of the repair machinery. The sciatic function index was introduced as a method to assess reinnervation after an experimental sciatic nerve lesion, and was adapted to the mouse model. The sciatic static index (SSI), is more simple and practical to perform, and is not so influenced by gait's velocity, but this method has not yet been adapted to the mouse model of sciatic lesion. We used 63 male Swiss mice (Mus musculus) to develop a formula to the sciatic static index in mice (SSIm). The animals were divided on three groups (control, transection and crush). They were evaluated at the preoperative and 7th, 14th, 21st, 28th, 35th and 42nd days postoperative by the ink track method (SFI), and by the acquisition of photographs of the plantar aspects of the injured and uninjured hind paws. The parameters evaluated were the 1-5 toe spread (TS), the 2-4 toe spread (ITS) and the distance between the tip of the third toe and the most posterior aspect of the paw (PL), on both methods. After verifying the temporal pattern of function, correlation and reproducibility of the measurements, we performed a multiple regression analysis using SFI values as dependent variable, and the TS, ITS and PL measured with the photo method as independent variables, and found the formula of the SSI for mice (SSIm). The three groups (control, transection and crush) had a characteristic pattern of dysfunction. The parameters measured in the ink and photo method had variable but significant correlations between them $(P<0.000)$, but photo method of measurement showed a better reproducibility. The correlation between SFI and SSIm showed a high correlation coefficient $(r=0.892$, $P<0.000$ ), and demonstrates that SSIm can be used as an alternative method to assess the functional status relative of sciatic nerve activity in mice.

(C) 2006 Published by Elsevier B.V.
\end{abstract}

Keywords: Functional tests; Mice; Sciatic nerve; Sciatic function index; Sciatic static index

\section{Introduction}

After an axonal lesion, there is an extensive degeneration of the distal segment, known as Wallerian Degeneration. Specially in the peripheral nervous system, the proximal stump, that is connected to the cell body, can regenerate to reinnervate the target organs. Although this process is often facilitated by a per-

\footnotetext{
* Corresponding author at: Departamento de Histologia e Embriologia, Universidade Federal do Rio de Janeiro, Cidade Universitária-Ilha do Fundão, Centro de Ciências da Saúde, Sala F012, Rio de Janeiro, RJ 21949-900, Brazil. Tel.: +55 21 25626431; fax: +552125626431.

E-mail address: martinez@ histo.ufrj.br (A.M.B. Martinez).
}

missive environment in the periphery (Stoll and Muller, 1999), some factors can impede normal return to function, such as the distance from injury site (Gordon et al., 2003), metabolic disturbances (Kennedy and Zochodne, 2005), age (Verdu et al., 2000) and type of lesion (Lundborg, 2004).

Strategies developed to improve peripheral nerve regeneration require quantitative approaches for evaluating functional outcome. The majority of such studies use rats as the chosen animal experimental model. In these studies, regeneration can be assessed by numerous methods, including histomorphometry, electrophysiology and gait analysis. Due to the fact that the sciatic represents the most used nerve in experimental approaches, some tests were developed to assess its function after injuries. 
The sciatic function index (SFI) is one of the most widely used forms of functional assessment. It compares parameters from the normal and experimental footprints by a mathematical formula, and provides information concerning the recovery of sensory-motor connections and cortical integration related to gait function and mediated by the sciatic nerve, without requiring terminal assessment. Since the SFI (DeMedinaceli et al., 1982) was introduced, it was modified several times in order to become simpler and more reliable (Bain et al., 1989; Carlton and Goldberg, 1986).

The sciatic static index (SSI), introduced by Bervar (2000), is another way of assessing recovery of function after sciatic injury in animal models. It uses the footprints, acquired when the animal is on a static position, and minimizes bias related to gait's velocity. Also, the SSI improves the acquisition of footprints, and is more repeatable and accurate then the SFI.

The SFI and the SSI were first developed to be used in rats (Bervar, 2000; DeMedinaceli et al., 1982). The SFI was adapted to be used in mice (Inserra et al., 1998), but the SSI, that is very simple and easy to perform, has not yet been adapted to this animal model. Furthermore, the use of mice in peripheral nerve regeneration studies is very common, because of the similarity of its biological processes with the human's, availability of transgenic models and because it is easier to manipulate. Nevertheless, functional tests in the mice model of sciatic nerve injury are poorly investigated. So, our goal was to develop the SSI formula for mice.

\section{Material and methods}

\subsection{Experimental animals and surgical procedure}

We used 63 male Swiss mice (Mus musculus), weighing $35-45 \mathrm{~g}$, from our animal facility. The study was in accordance with the rules of the Committee for Ethics in Animal Experimentation of the Gonçalo Moniz Research Center/Oswaldo Cruz Foundation, Brazil. The animals were housed in individual cages with food and water ad libitum and a cycle of $12 \mathrm{~h} \mathrm{light} / 12 \mathrm{~h}$ dark. Animals were deeply anesthetized with ketamine $(0.1 \mathrm{~mL} / \mathrm{mg})$ and xylazine $(0.2 \mathrm{~mL} / \mathrm{mg})$ and then submitted to asepsis and trichotomy of the right gluteus region. After a longitudinal incision, the sciatic nerve was either only exposed and isolated from the adjacent tissues (control group) $(n=21)$, crushed at $1 \mathrm{~cm}$ from the spinal cord with a non-serrated needle holder, with an uniform pressure applied for $30 \mathrm{~s}$ (crush group) $(n=21)$, or transectioned at the sciatic notch, with the removal of a $2 \mathrm{~mm}$ gap (transection group) $(n=21)$. The muscle and skin were sutured by layers.

\subsection{Functional tests}

In the previous day and on 7th, 14th, 21st, 28th, 35th and 42 nd days post lesion, the animals were evaluated to obtain three footprint parameters- the distance between the first and fifth toes: toe spread (TS), the distance between the second and fourth toes: intermediary toe spread (ITS) and the distance between

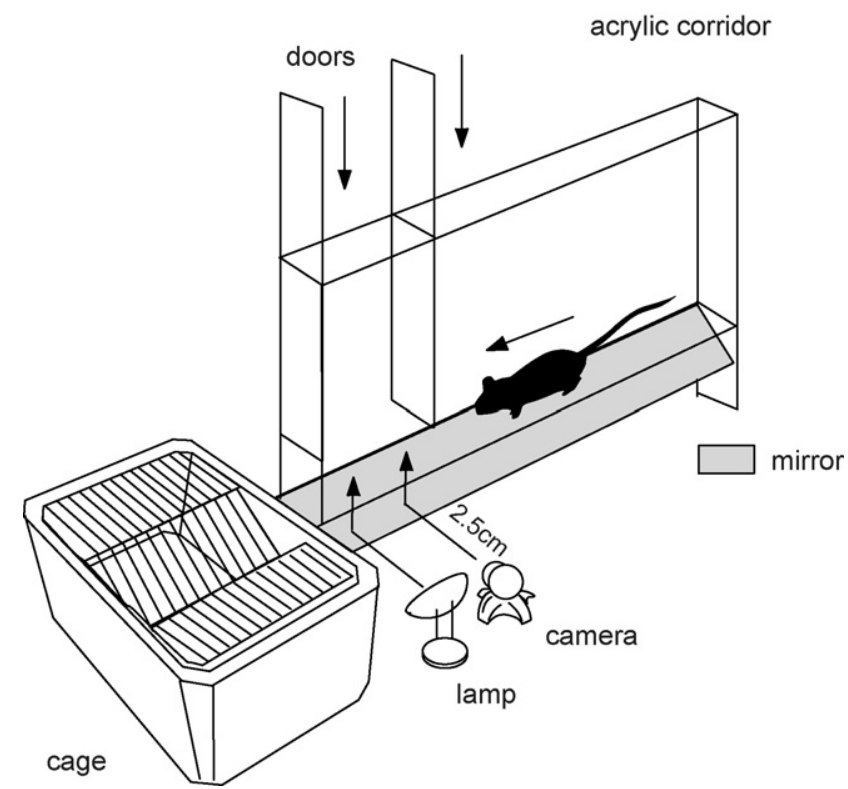

Fig. 1. Apparatus to gait photo assessment. The animals were induced to walk in its cage direction, through a transparent acrylic corridor, with a mirror at $45^{\circ}$ underneath the floor. At the end of the track, two doors were closed and, when the animal stopped, the photos were acquired with a Web Cam positioned $2.5 \mathrm{~cm}$ apart from the mirror, under illumination of a $50 \mathrm{~W}$ lamp. After the photos were acquired, the animals were permitted to go to the cage's grid and transported inside.

the tip of the third toe and the most posterior part of the foot in contact with the ground: print length (PL).

The assessment was carried out in two ways. First, the animals were disposed on an acrylic corridor $(48 \mathrm{~cm} \times 4.5 \mathrm{~cm})$ with a mirror placed underneath the apparatus at an angle of $45^{\circ}$ (Varejão et al., 2001; Westerga and Gramsberg, 1990). A $50 \mathrm{~W}$ lamp, with the focus directed to the mirror, illuminated the corridor (Fig. 1). The animal's cage was placed at the end of the corridor to induce the animal to walk in this direction, and before it got into it, the corridor was closed (Fig. 1). When the animals stopped, at the end of the corridor, three photos were acquired with a Web Cam (Drop Cam 100, Go Tec, China), positioned $2.5 \mathrm{~cm}$ from the mirror, and the parameters' means of the footprint assessed with an image analysis software (Image J, National Institute of Health, USA), calibrated with a scanned pachymeter. The TS and ITS were defined with the most lateral and medial edges of the proper toes, and the PL with the most anterior edge of the third toe and the most posterior portion in contact with the ground, where the limits of the paw became less clear, pale and discolored (Fig. 2).

After the photos were obtained on the acrylic corridor, the animals were disposed on a similar dispositive, made of wood and lined on the bottom with a sheet white paper. The animals were immobilized and had the hind limb paws painted with black China's ink, until the rear portion of the foot. Then they were allowed to walk along the corridor and the footprints were registered. For each animal, a mean of three pairs of footprints, each from the same gait cycle, was used in the assessment. These parameters were measured with a pachymeter, and the TS and ITS measurements followed the same criteria as the photo ones. 


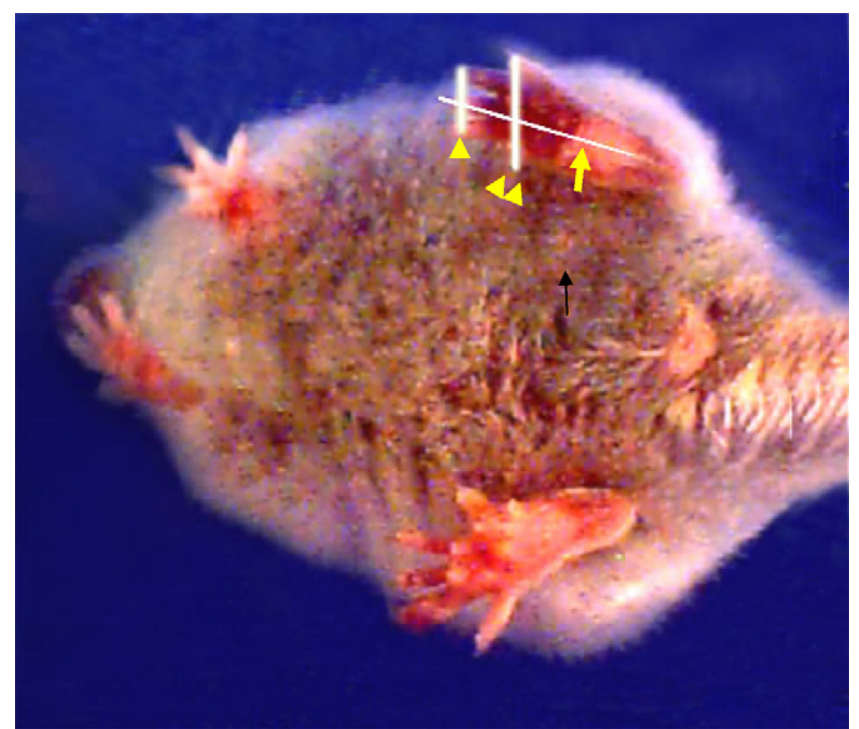

Fig. 2. Photograph showing the plantar aspect of a mouse. Bars show 1-5 toe spread (TS) (arrow head), 2-4 intermediary toe spread (ITS) (double arrow head) and print length (PL), estimated as the distance from the tip of the third toe to the most posterior aspect of the paw in contact with the floor (arrow). Three photos were acquired from each animal and the mean TS, ITS and PL achieved to assess gait function.

For the PL we considered the tip of the third toe and the most posterior aspect of the footprint.

The factors for each parameter (TS, ITS and PL) were calculated with the formula: injured - uninjured/uninjured values (e.g. TSF (toe spread factor) $=$ TS experimental - TS normal/TS normal), and the SFI with the one conceived by Inserra et al. (1998), where SFI $=118.9 \times \mathrm{TSF}-51.2 \times \mathrm{PLF}-7.5$.

\subsection{Data analysis}

Statistical analysis was performed in various steps. First, we assessed the degree of variability on function per group, across the 6-week period of experiment, using Friedmann's paired analysis of variance on the SFI values. The second step was to find correlation between each footprint parameter and also their factors, obtained with the two methods (photo and ink), using Pearson's correlation index. Third, we verified the reproducibility of the measurements, using ANOVA to compare variability of each parameter between the two forms of assessment (ink and video), across the period of experiment. After a curve fit test, to verify if the values were distributed on a linear disposition, we used multiple linear regression analysis. It was considered as dependent variable the values of the SFI through all the period, and as independent variables the TS, ITS and PL factors, obtained with the photo method. We defined the functional losses as zero preoperatively and -100 postoperatively and, using regression analysis, generated the adequate SSI formula for mice (SSIm). Then we assessed correlation between SSIm and SFI, pooling again all the three groups (control, transection and crush), using Pearson's correlation index. It was accepted the significance level of $5 \%(P<0.05)$. The analysis was carried out with the OPENSTAT 4.0 statistical package.

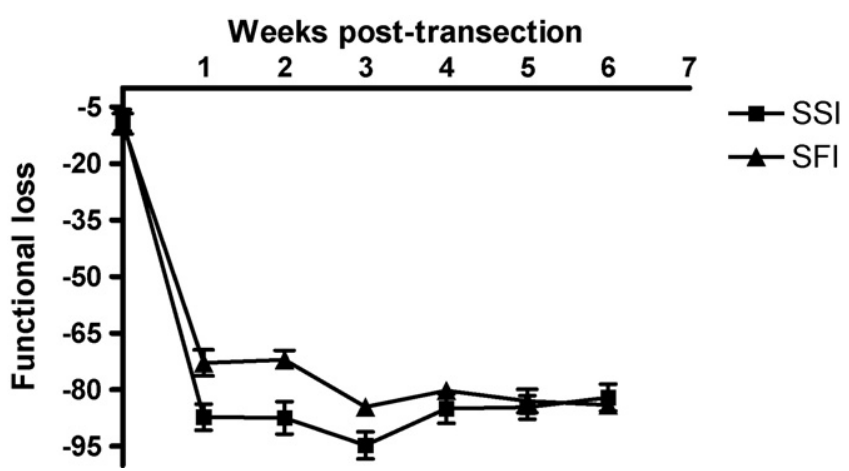

Fig. 3. Comparison of the recovery determined by sciatic function index (SFI) $(\boldsymbol{\Delta})$ and sciatic static index for the mouse (SSIm) (ם) in the transection group. The graph shows function during the 6-week period of experiment, assessed by the SFI and SSIm. Static sciatic index for the mouse seems to be a little more sensitive to transection then SFI, showed by a more prominent decrease of function on the first 3 weeks post lesion. Data are presented as mean \pm S.E.M.

\section{Results}

The animals were analyzed weekly, from the day immediately before surgery, and the analysis was carried out for 6 weeks. Statistical tests using Friedmann analysis of variance on SFI values, showed no significant shift on the control group function, during the 6 weeks period of experiment $(F=6.454$, d.f. $=6, P=0.3743)$. Transection group followed a different pattern, with a marked decrease on function at the first week post-lesion, and maintained this status until the end of the experiment $(F=50.526$, d.f. $=6, P<0.0001)$ (Fig. 3). Crush group also showed the same decrease on function in the first week $(F=60.00$, d.f. $=6, P<0.0001)$, but recovering normal gait at the third week (Fig. 4). Two animals were excluded from both, crush and transection groups, due to autotomy.

Correlation between ink and photo measurements, using Pearson's index, was significant for all the parameters $(P<0.000)$. Although some of them were low (uninjured TS and PL, and injured ITS), the remaining showed mild to high correlation (Table 1). The analysis of the parameter's reproducibility showed coefficients of variations ranging from $8.73 \%$ (TS, uninjured side, ink method) to $28.57 \%$ (ITS, uninjured

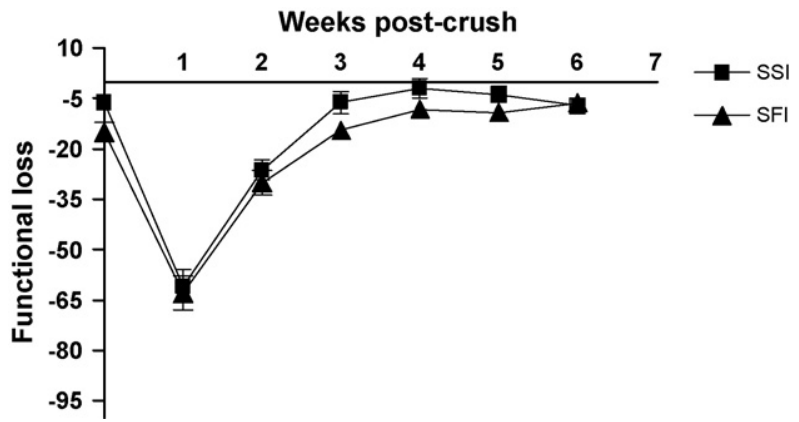

Fig. 4. Comparison of the recovery determined by sciatic function index (SFI)

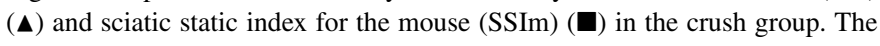
graph shows function during the 6-week period of the experiment, assessed by the SFI and SSIm. Both methods show similar pattern, with marked decrease of function on the first week, and return to normal on the third or fourth weeks. Data are presented as mean \pm S.E.M. 
Table 1

Correlation between photo vs. ink measurements of the footprint variables (TS, ITS and PL) and their calculated factors

\begin{tabular}{llll}
\hline Parameter & $\begin{array}{l}\text { Degrees of } \\
\text { freedom (d.f.) }\end{array}$ & $\begin{array}{l}\text { Correlation } \\
\text { coefficient }(r)\end{array}$ & $\begin{array}{l}\text { Significance } \\
\text { level }(P \text {-value })\end{array}$ \\
\hline 1-5 toe spread (TS) & & & \\
$\quad$ Uninjured hind foot & 413 & 0.363 & $<0.000$ \\
$\quad$ Injured hind foot & 413 & 0.882 & $<0.000$ \\
TS factor & 413 & 0.875 & $<0.000$ \\
2-4 toe spread (ITS) & & & \\
$\quad$ Uninjured hind foot & 413 & 0.609 & $<0.000$ \\
$\quad$ Injured hind foot & 413 & 0.213 & $<0.000$ \\
$\quad$ ITS factor & 413 & 0.608 & $<0.000$ \\
Print length (PL) & & & \\
$\quad$ Uninjured hind foot & 413 & 0.234 & $<0.000$ \\
$\quad$ Injured hind foot & 413 & 0.540 & $<0.000$ \\
$\quad$ PL factor & 413 & 0.488 & $<0.000$
\end{tabular}

Factor $=$ injured - uninjured/uninjured.

Table 2

Reproducibility of the footprint parameters

\begin{tabular}{|c|c|c|c|c|}
\hline \multirow[t]{2}{*}{ Parameter } & \multirow{2}{*}{$\begin{array}{l}\text { Degrees of } \\
\text { freedom } \\
(\text { d.f. })^{\mathrm{a}}\end{array}$} & \multicolumn{2}{|c|}{$\begin{array}{l}\text { Average coefficient } \\
\text { of variation }(\%)\end{array}$} & \multirow{2}{*}{$\begin{array}{l}\text { Most repeatable } \\
\text { technique } \\
(P \text {-value })\end{array}$} \\
\hline & & Ink & Photo & \\
\hline \multicolumn{5}{|l|}{$1-5$ toe spread (TS) } \\
\hline Uninjured hind foot & 1 & 8.73 & 10.38 & Ink $(P<0.01)$ \\
\hline Injured hind foot & 1 & 29.41 & 8.82 & Photo $(P<0.00)$ \\
\hline \multicolumn{5}{|l|}{ 2-4 toe spread (ITS) } \\
\hline Uninjured hind foot & 1 & 18.22 & 16.33 & Photo $(P<0.00)$ \\
\hline Injured hind foot & 1 & 26.19 & 28.57 & Ink $(P<0.00)$ \\
\hline \multicolumn{5}{|l|}{ Print length (PL) } \\
\hline Uninjured hind foot & 1 & 13.9 & 9.32 & Photo $(P<0.05)$ \\
\hline Injured hind foot & 1 & 27.76 & 11.10 & Photo $(P<0.00)$ \\
\hline
\end{tabular}

a Between groups.

side, ink method). Ink and photo methods of acquiring footprint parameters were always different, with photo method being more reproducible than ink method on almost all conditions. Ink method was more repeatable only for the uninjured TS and ITS measurements. Injured side had, almost always, bigger coefficients of variation (Table 2). Toe spread measurements seemed to be the most accurate measurements, with coefficients of variation ranging from 8.73 to $10.38 \%$.

In order to find the SSI formula for mice (SSIm), we performed multiple linear regression using as dependent variable SFI values, obtained through all period of experiment, pooling the three groups of animals (control, transection and crush). To minimize errors, we used only those values that were between 10 and -100 . Fifteen values were excluded because they were not in this range. The independent variables were the three factors obtained in the photo assessment (FTS, FITS and FPL). A curve fit test was performed to verify if they had a linear distribution. All of the three achieved a significance level, with the $F$ test being stronger in the FTS group $(F=1445.22, P<0.000)$, followed by the FITS $(F=450.72, P<0.000)$ and the FPL $(F=231.96$, $P<0.000)$.

Multiple linear forward stepwise regression analysis was performed, and showed toe spread factor as the major function predictor, representing almost $78 \%$ of the variations $\left(R^{2}=0.778\right.$, $F=1445.22, P<0.000)$. Intermediary toe spread factor did not change the estimative of function, and PLF generated minor but significant changes. When PLF was added to FTS, they turned to represent almost $80 \%$ of the variations $\left(R^{2}=0.796\right.$, $F=51130.33, P<0.000$ ) (Table 3 ). Based on this model we found the formula SSIm $=101.3 \times \mathrm{TSF}-54.03 \times \mathrm{PLF}-9.5$ (Table 4). The $F$ value for the equation was 798.728 , significant at the $P<0.000$ level.

The next step was to validate the new formula finding its correlation with the previous SFI index. Pearson's index was performed to correlate all SFI index values with the ones now obtained using the SSIm formula, pooling again all the groups across the period of experiment. We found a high and significant correlation between the values obtained by the two formulae $(r=0.892$, two-tailed, $P<0.000)$. Static sciatic index for mice generated a similar pattern of function compared to SFI on the three groups analyzed. Little differences were seen, like on the first week post lesion, where SFI values did not decrease so much as SSIm on the transection group (Fig. 3). These differences where not seen when SFI and SSIm where compared on the crush group (Fig. 4)

\section{Discussion}

The search for a practical, reproducible and sensitive test to analyze function recovery after a peripheral nerve lesion is very important because these methods allow the quantitative determination of the recovery process, which relies on the functional reinnervation of the target organs. Bona fide quantitative methods are needed for the assessment of rehabilitation. Many tests were developed and adapted to assess sensory-motor recovery in the rat model of sciatic nerve injury (Schiaveto de Souza et al., 2004; Varejão et al., 2004a). Although this rodent is the most widely used animal model in studies of peripheral nerve regeneration, the mouse is not only well characterized genetically,

Table 3

Linear regression model, showing the power of each factor on the final SSI formula for mice

\begin{tabular}{|c|c|c|c|c|c|c|c|c|c|}
\hline \multirow[t]{2}{*}{ Factor } & \multirow[t]{2}{*}{$R$} & \multirow[t]{2}{*}{$R^{2}$} & \multirow[t]{2}{*}{ Adjusted $R^{2}$} & \multirow[t]{2}{*}{ S.E. } & \multicolumn{5}{|c|}{ Change statistics } \\
\hline & & & & & $R^{2}$ change & $F$ change & d.f.1 & d.f. 2 & Sig. $F$ change \\
\hline TS factor & $0.883^{\mathrm{a}}$ & 0.779 & 0.778 & 17.74395 & 0.779 & 1445.217 & 1 & 410 & $P<0.000$ \\
\hline
\end{tabular}

\footnotetext{
${ }^{\text {a }}$ Predictors: (constant), ftsV.

b Predictors: (constant), ftsV, fplV.
} 
Table 4

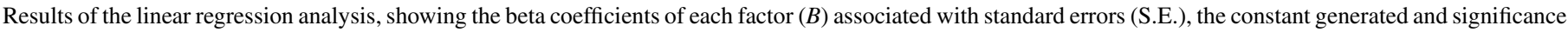
level of the estimations

\begin{tabular}{|c|c|c|c|c|c|}
\hline \multirow[t]{2}{*}{ Footprint factor } & \multicolumn{2}{|c|}{ Unstandardized coefficients } & \multirow[t]{2}{*}{ Standardized coefficients, beta } & \multirow[t]{2}{*}{$t$} & \multirow[t]{2}{*}{ Significance level ( $P$-value) } \\
\hline & $B$ & S.E. & & & \\
\hline Constant & -9.949 & 1.034 & & -9.618 & $<0.000$ \\
\hline TS factor & 101.29 & 5.29 & 0.765 & 19.146 & $<0.000$ \\
\hline ITS factor & 5.64 & 5.78 & 0.036 & 0.975 & 0.330 \\
\hline PL factor & -54.03 & 9.13 & -0.159 & -5.918 & $<0.000$ \\
\hline
\end{tabular}

but also easier to manipulate and maintain. Gait assessment is expressive to represent regeneration after peripheral nerve lesions, since it integrates sensory and motor fibers on a specialized pattern of movement. The SFI was adapted to the mouse model of sciatic lesion by Inserra et al. (1998). In this work, only the print length (PL) and the toe spread (TS) were considered relevant to assess function variations. To register the footprints, the use of China's ink and paper may be a simple method to allow clear identification of the footprint parameters and permits various gait cycles to choose, but it may stress the animals and is time consuming. Also, there are some negative factors that can introduce bias in the study, such as foot contractures, autotomy, excrements on the corridor (Bain et al., 1989; DeMedinaceli et al., 1982; Varejão et al., 2004a) and variations due to gait velocity (Walker et al., 1994a).

The SSI, developed by Bervar (2000), seems to be easier and faster to be acquired and analyzed, generating considerably less stress to the animals. In our method we found that the images turned out to be very clear, facilitating the measurement of the parameters involved. Besides, it requires only a low cost dispositive, that includes a device with transparent bottom, a photographic camera and an imaging processing system, and works well under low illumination, i.e. approximately $25 \%$ of the power used by Bervar (2000), presumably reducing the animal's stress, as rodents are light aversive.

The three groups on our experiment showed an expected pattern of function during the 6 weeks period, similar to other studies in rats (Bervar, 2000; Grasso et al., 2004; Varejão et al., 2004b) and mice (Fukuyama et al., 2000; Islamov et al., 2002). Ink and photo methods used to acquire footprint parameters had, always, different reproducibility. Photo measurements were more accurate, presenting most of the time lower coefficients of variation than ink method. The bigger variability on the ink method may be due to difficulties with smeared footprints, contractures and variations on gait velocity, already seen by others (Dellon and Mackinnon, 1989; Jihad et al., 1989; Varejão et al., 2001, 2004b; Walker et al., 1994a). Also, the dynamic procedure to achieve footprints has sometimes to be repeated, because of the low quality of some prints. This repetition stresses the animals and can contribute to greater variations on the measurements. The photos used on the static analysis minimize most of these problems, because the researcher can wait for the moment where animals are stopped to acquire the images. There is no bias due to smeared footprints, as we can see paws as they are, or to gait velocity variations, because the animals are stopped. Maybe variations due to weight or sex (Dellon and Dellon, 1991;
Parker and Clark, 1990) can also be minimized with the static method, because the animal is lying on its posterior paws, dissipating mechanical charges. Sometimes, excrements or forced adduction of the lateral toes by the cages' walls interfered with the measurements. On these situations, we cleaned the acrylic dispositive or gently moved the animals to another position. We consider that it is very important to adapt the animals to the procedure. When it was not a new situation, they seemed to be quieter, stopping just at the end of the corridor, and maintaining a static position.

The formula developed for the SSIm, shows the big influence of the TS as a measure of gait dysfunction due to sciatic nerve lesion. This parameter represents the majority of alterations and may be used alone as an indicative of the lesion's stage, as have been demonstrated by other investigators (Bervar, 2000; Walker et al., 1994a). Intermediary toe spread, following the same pattern of the SFI for mice (Inserra et al., 1998), did not have a significant role on demonstrating dysfunction, probably because it is very similar on the crush and control groups, or because of its big variations. The PL gave a small but significant contribution to the formula. This parameter expresses the ability of the soleous and gastrocnemious muscles to maintain the heel elevated during walking in rodents. As these muscles are innervated by the sciatic nerve, when impaired they cannot maintain the normal ankle angle, letting a bigger portion of the paw to be in contact with the floor. Although the PL may be very variable, due to the difficulty to establish its posterior aspect, its significance has also been demonstrated in the ink track method, which generated the SFI formula for the mouse (Inserra et al., 1998).

The high correlation between the SFI and SSIm $(r=0.892$, $P<0.000)$ validates the use of the static analysis to assess sciatic nerve dysfunction after lesions in mice. Afterwards, SSIm is much more simple to perform, because it does not require the use of paint or other resources that can confound the measurement of each parameter. Also, as the animal does not need to walk, variations due to gait velocity and changes on the direction are not involved.

Further studies should investigate the use of the SSIm to assess different types of lesions, like transection and repair or tubulization. Also it is necessary to assess the use of SSIm to evaluate chronic injuries, as the SFI does not seem to be capable to demonstrate functional status in these situations due to the presence of contractures. In this situation, the abnormal gait pattern may influence the tail to smear ink or retain it in the claws, generating difficulties to interpretation of data (Dellon 
and Mackinnon, 1989). Although this does not happen on photo assessments, contractures are important consequences of nerve injury, and studies should be developed to assess its influence on SSIm. Correlations between the SSIm and histomorphometric measurements should also be performed, as Jihad et al. (1989) showed that function assessed by the SFI may not be correlated with morphological findings.

\section{Acknowledgement}

This research was supported by grants from Fundação Bahiana para Desenvolvimento das Ciências, CAPES, FAPESB, FAPERJ and CNPq. We would like to thank to Israel Souza, Marcos Aguiar and Maurício Cardeal for statistical analysis support and Tarcísio de Sá Cardoso for the ilustrations.

\section{References}

Bain JR, Mackinnon SE, Hunter DA. Functional evaluation of complete sciatic, peroneal and posterior tibial nerve lesions in the rat. Plast Reconstr Surg 1989;83(1):129-38.

Bervar M. Video analysis of standing — an alternative footprint analysis to assess functional loss following injury to the rat sciatic nerve. J Neurosci Meth 2000;102(2):109-16.

Carlton JM, Goldberg NH. Quantitative integrated muscle function following reinnervation. Plast Reconstr Surg 1986;37:611-2.

Dellon ES, Dellon AL. Functional assessment of neurological impairment: track analysis in diabetic and compression neuropathies. Plast Reconstr Surg 1991;88:686-94.

Dellon AL, Mackinnon SE. Sciatic nerve regeneration in the rat. Validity of walking track assessment in the presence of chronic contractures. Microsurgery 1989;10(3):220-5.

DeMedinaceli L, Freed WJ, Wyatt RJ. An index of the functional condition of rat sciatic nerve based on measurements made from walking tracks. Exp Neurol 1982;77(3):634-43.

Fukuyama R, Ohta M, Ohta K, Saiwaki T, Fushiki S, Awaya A. A synthesized pyrimidine compound, MS-818, promotes walking function recovery from crush injury of the sciatic nerve through its indirect stimulation of Schwann cells. Restor Neurol Neurosci 2000;17(1):9-16.
Gordon T, Sulaiman O, Boyd JG. Experimental strategies to promote functional recovery after peripheral nerve injuries. J Peripher Nerv Syst 2003;8:236-50.

Grasso G, Sfacteria A, Brines M, Tomasello F. A new computed-assisted technique for experimental sciatic nerve function analysis. Med Sci Monit 2004;10(1):BR1-3.

Inserra MM, Bloch DA, Terris DJ. Functional indices for sciatic, peroneal, and posterior tibial nerve lesions in the mouse. Microsurgery 1998;18(2):119-24.

Islamov RR, Hendricks WA, Jones RJ, Lyall GJ, Spanier NS, Murashov AK. 17Beta-estradiol stimulates regeneration of sciatic nerve in female mice. Brain Res 2002;943(2):283-6.

Jihad M, Shenaq MD, Saleh M, Shenaq MD, Melvin S. Reliability of sciatic function index in assessing nerve regeneration across a $1 \mathrm{~cm}$ gap. Microsurgery 1989;10(3):214-9.

Kennedy JM, Zochodne DW. Impaired peripheral nerve regeneration in diabetes mellitus. J Peripher Nerv Syst Jun 2005;10(2):144-57.

Lundborg G. Nerve injury and repair-regeneration, reconstruction and cortical repair. Philadelphia: Elsevier/Churchill Livingstone; 2004.

Parker AJ, Clark KA. Gait topography in rat locomotion. Physiol Behav 1990;48:41-7.

Schiaveto de Souza A, da Silva CA, Del Bel EA. Methodological evaluation to analyze functional recovery after sciatic nerve injury. J Neurotrauma 2004;21(5):627-35.

Stoll G, Muller HW. Nerve injury, axonal degeneration and neuronal regeneration: basic insights. Brain Pathol Apr 1999;9(2):313-25.

Varejão ASP, Meek MF, Ferreira AJA, Patrício JAB, Cabrita AMS. Functional evaluation of peripheral nerve regeneration in the rat: walking track analysis. J Neurosci Meth Jul 2001;108(1):1-9.

Varejão ASP, Melo-Pinto P, Meek MF, Filipe VM, Bulas-Cruz J. Methods for the experimental functional assessment of rat sciatic nerve regeneration. Neurol Res 2004a;26(2): 186-94.

Varejão ASP, Cabrita AM, Meek MF, Bulas-Cruz J, Melo-Pinto P, Raimondo S, et al. Functional and morphological assessment of a standardized rat sciatic nerve crush injury with a non-serrated clamp. J Neurotrauma 2004b;21(11):1652-70.

Verdu E, Ceballos D, Vilches JJ, Navarro X. Influence of aging on peripheral nerve function and regeneration. J Peripher Nerv Syst Dec 2000;5(4):191-208.

Walker JL, Evans JM, Meade P, Resig P, Sisken BF. Gait-stance duration as a measure of injury and recovery in the rat sciatic nerve model. J Neurosci Meth 1994a;52(1):47-52.

Westerga J, Gramsberg A. The development of locomotion in the rat. Brain Res Dev Brain Res 1990;57(2):163-74. 